\title{
AGRESIONES SEXUALES INFANTO-JUVENILES: UNA APROXIMACIÓN A VÍCTIMAS DE AGRESORES MENORES DE EDAD*
}

\begin{tabular}{l} 
CHILD SEXUAL ABUSE: AN APPROACH TO VICTIMS WHO \\
ARE MOLESTED BY OTHER MINORS \\
\hline
\end{tabular}

Recibido: 25 de Junio del 2013 | Aceptado: 10 de Diciembre del 2013

\author{
CAROLINA PUYOL WILSON ${ }_{1}$; MARÍA ISABEL SALINAS CHAUD 2 \\ (UNIVERSIDAD DE CHILE, Santiago de Chile, Chile)
}

\begin{abstract}
RESUMEN
El presente estudio exploró las características de las dinámicas abusivas y vulnerabilidad de víctimas infanto-juveniles de agresiones sexuales cometidas por otro niño/a y/o adolescente. La investigación se realizó desde un paradigma cualitativo por medio de la metodología de análisis de contenido de documentos, consistentes en 76 informes de evaluaciones periciales psicológicas a víctimas tanto penales como proteccionales realizadas en un Centro de Diagnóstico Ambulatorio de la Región Metropolitana. Dentro de los principales resultados, no se observa ninguna dinámica abusiva sin vínculo previo entre víctima y victimario, ni tampoco fue posible constatar existencia de agresores de menor edad y/o desarrollo evolutivo que la víctima, caracterizándose estas últimas de manera predominante en una etapa de niñez.

Las principales conclusiones corresponden a la importancia de los factores de vulnerabilidad en la víctima, como factor posibilitador de la condición de asimetría de poder entre esta y su agresor. Asimismo, el tipo de vínculo diferenciará notoriamente las características de la dinámica abusiva.
\end{abstract}

PALABRAS CLAVE: Agresión sexual, dinámicas abusivas, vulnerabilidad.

\section{ABSTRACT}

This study explores the characteristics of abusive dynamics and the vulnerability of child and adolescent victims of sexual aggressions which have been committed by other minors. The research was conducted from a qualitative paradigm using the methodology of content analysis of documents. These documents were 76 reports of both criminal forensic and psychological evaluations of victims, both those reporting crimes and those under legal protection due to earlier crimes. These reports were taken at a walk-in diagnosis center in Santiago, Chile. The results show no abusive dynamics between attacker and victim without a previously existing relationship. Neither was it possible to verify existence of offenders of lesser age and/or evolutionary development that their victims. Characteristically, the aggressor was an adolescent and the victim a child. These findings support the importance of vulnerability in the victim as a factor which facilitates the asymmetry of power between aggressor and victim. Additionally, the nature of the relationship between the victim and his/her attacker will determinate characteristics of the upcoming abusive dynamics.

KEY WORDS: Sexual abuse, sexual abuse dynamics, vulnerability.

\footnotetext{
Este artículo es parte de la tesis de magíster para la obtención del título de Magíster en Intervención Psicojurídica y Forense de la Universidad Diego Portales de la primera autora, la cual se desarrolló bajo la supervisión, dirección y patrocinio de la segunda autora.

1. Magíster. Psicóloga del Poder Judicial en Proyecto de implementación de sala Gesell en Tribunales de Familia, Santiago de Chile, Chile. E-mail: carolina.puyol.wilson@gmail.com 2. Doctora en Psicología y profesora asistente del Departamento de Psicología de la Universidad de Chile, Santiago de Chile, Chile. E-mail: misrpp@hotmail.com
} 


\section{INTRODUCCIÓN}

Las agresiones sexuales infanto-juveniles se han caracterizado usualmente por dos elementos, primero que estas son cometidas por adultos, y además que estos son predominantemente de sexo masculino. Por tanto, el desarrollo y estudio de este fenómeno, se ha centrado tanto a nivel victimológico como criminológico en las variables antes señaladas.

No obstante, en el último tiempo ha existido una preocupación y un cambio de foco respecto de otros tipos de agresores posibles: mujeres, y niños/as y adolescentes, que han logrado una mayor visibilidad y relevancia social, que anteriormente quedaba oculta tras la cifra negra de este tipo de delitos.

Especialmente, en el último grupo ha existido un aumento y mayor notoriedad de acciones delictuales principalmente en contra de la propiedad y de las personas, dado en algunos casos la magnitud de violencia ejercida y/o inicio precoz. De este modo, los imputados entre 14-17 años han tenido un aumento del $19,5 \%$ en los últimos 5 años (Fundación Paz Ciudadana, 2011) y sólo en la Fiscalía Regional Metropolitana Centro Norte (2010) ha existido un aumento promedio de $67 \%$ de la frecuencia de denuncias de agresiones sexuales infantojuveniles cometidas por adolescentes sólo en el lapso de dos año, mientras que a nivel país, se ha registrado un total de 3.237 imputados de delitos sexuales tras el inicio de la Reforma Penal desde su inicio en el año 2000 (Correa et. al., 2009).

Las cifras antes expuestas, tienen concordancia con los hallazgos disciplinares producto del trabajo con agresores sexuales, donde se ha identificado que dichas transgresiones se desarrollan $y$ ocurren desde la adolescencia 0 incluso antes, manteniendo un aumento conductual escalar en términos de gravedad (Horton, 1996), por tanto la detección de dicho tipo de conductas en las etapas de infancia y adolescencia, corresponden a un potencial de desarrollo de un tipo particular de personalidad, donde se instaura un interés sexual desviado (Asenjo, 2003 y Wolf, 1988), a partir de experiencias tempranas. En esta misma línea, algunos datos obtenidos por medio de investigaciones internacionales con agresores sexuales adultos, dan cuenta de que más del $50 \%$ de estos cometen su primera agresión antes de los 16 años y sólo $35 \%$ de ellos habrían contado con un registro oficial que documentara dicho acto delictual en sus etapas de adolescencia (Correa et. al., 2009; Díaz, 2003).

Por otro lado, estudios internacionales como los de Johnson (1988), realizado con niños/as y adolescentes que habían cometido transgresiones sexuales, exponen que la edad promedio de los agresores fue de 8 años y 9 meses cuando las victimizaciones fueron llevadas a cabo, mientras que la edad promedio de las víctimas fue de 6 años y 9 meses. Igualmente, se destaca que la coerción fue el mecanismo de sometimiento utilizado en todos los casos revisados, donde también existiría un vínculo entre víctima y victimario, característica que también habría sido revelada por otros estudios (Díaz, 2003).

De este modo, la muestra de los estudios de Johnson (1988) se caracterizó por poseer un $47 \%$ de casos con vínculo intrafamiliar, con predominancia de relación fraterna. Por otro lado, el promedio de víctimas por agresor fue de 2,1 con una variación de 1 a 7 víctimas, destacando a su vez la existencia de disfuncionalidad familiar en la mayoría de los casos, aspecto que también habría sido denotado en procesos de rehabilitación de agresores sexuales adolescentes de programas chilenos asociados a la Policía de Investigaciones (Escaff, 2009). 
Otros trabajos internacionales, como los de Berlinger y Rawlings (en Horton, 1996) han generado un modelo descriptivo del continuo en que ocurren las transgresiones sexuales entre niños/as y/o adolescentes, con el objeto de identificar desde las conductas exploratorias hasta aquellas de tipo abusivas, en especial cuando la asimetría no es clara al no existir un diferente nivel evolutivo (Volbert, 2005). De este modo, Berlinger y Rawlings (en Horton, 1996) manifiestan que a pesar de la poca claridad de la asimetría, las transgresiones sexuales cometidas por un niño/a y/o adolescente son desarrolladas de manera paulatina y en un continuo de menor a mayor gravedad de conductas sexuales posibles: inapropiadas, precoces y/o coercitivas. En esta producción teórica se destaca la forma de sometimiento utilizado como elemento diferenciador al momento de realizar la conducta de agresión sexual.

De este modo, si bien existen avances en la temática en relación a estudios, como los antes vistos, y a programas de intervención con agresores adolescentes (Venegas, 2005), ambos se encuentran desde la lógica del victimario, y en el caso chileno, se limitan a una conceptualización de imputabilidad adolescente, lo que se torna como una mirada reduccionista $\mathrm{e}$ invisibiliza no sólo a aquellos que no son susceptibles de sanción penal, sino también a las víctimas que no tendrán una mirada diferenciada en relación a este fenómeno. Por lo que, se ha ignorado conocer el fenómeno desde una mirada victimológica, que permita comprender la dinámica abusiva y las características particulares de las víctimas de esta configuración de transgresión sexual.

Esto último se debe a que las dinámicas de victimización sexual comprenden diversos elementos que se retroalimentan entre sí y que diferenciarán una agresión de otra. De esta forma, Salinas (2006) manifiesta que las agresiones sexuales se configurarán diferencialmente a partir del vínculo entre víctima y victimario, frecuencia de victimización, formas de sometimiento, características de la develación y percepción de daño. Estos elementos dinámicos, también interactuaran con otros de carácter individual, como la vulnerabilidad puesto que posicionan a la víctima en una posición de inequidad (Herrera y Campero, 2002).

Asimismo, la vulnerabilidad es un elemento diferenciador, ya que se encuentra "en función de distintos tipos de delitos ya que una persona puede ser extremadamente vulnerable, pero muy resistente para otros" (Guerra y Canessa, 2007, Pág. 121). Para comprender la vulnerabilidad de una manera integral, Esbec y Gómez-Jarabo (2000), proponen la existencia de 5 elementos que la configuran: la vulnerabilidad general, componentes biológicos, rasgos de personalidad, recursos sociales, y factores biográficos.

De esta manera, la exploración tanto dinámica como individual en las víctimas de este fenómeno es de gran utilidad al permitir descubrir los factores asociados a este tipo de victimización, no sólo en torno a los elementos estáticos, sino que también respecto de las circunstancias interaccionales que favorece la comisión del delito. Esta mirada victimológica, permite entonces comprender qué condiciones posibilitan el establecimiento de asimetría especial entre víctimas y victimario, logrando establecer una dinámica de sumisión y por ende una vulnerabilidad propia que no se condice necesariamente con el paradigma del adultismo en la sumisión sexual. Por tanto, la sistematización de estos factores ha de contribuir entonces a reflexiones $y$ propuestas en torno a políticas de prevención, y a lineamientos respecto de los quehaceres de diagnóstico e intervenciones reparatorias especializadas.

De este modo, el presente estudio tuvo dos objetivos generales, consistentes en la 
descripción de las características de vulnerabilidad de las víctimas infantojuveniles que han sido agredidas sexualmente por parte de otro niño/a y/o adolescente, como también la exploración de las características y/o diferencias en las dinámicas abusivas de este fenómeno. Para el primer objetivo, se indagará en las características personales y socio-familiares de las víctimas, mientras que para el segundo objetivo se describirán las dinámicas abusivas principalmente en dos grupos, el primero cuando tanto víctima como victimario tengan un mismo desarrollo evolutivo, y el segundo cuando estos sean dispar.

\section{MÉTODO}

La presente investigación se realizó desde un paradigma cualitativo, con el propósito de profundizar en las características de las víctimas infanto-juveniles de agresiones sexuales por parte de otro niño, niña 0 adolescente, y que a su vez permitiera el desarrollo de un proceso de investigación flexible donde emerjan nuevas hipótesis de dicho proceso dialéctico (Denzin y Lincoln, 2005).

El alcance de la presente investigación fue descriptivo, puesto que su objetivo fue precisar características de las dinámicas abusivas y de vulnerabilidad de la muestra escogida. En esta misma línea, el diseño de la investigación correspondió a uno de carácter no experimental al no existir una manipulación deliberada de variables como tampoco de una asignación aleatoria de los sujetos a grupos de investigación, ante la imposibilidad práctica y ética del tipo de sujetos y temática que se exploró y analizó en la presente investigación.

$Y$ por otro lado, el diseño no experimental correspondió a uno de tipo transeccional, donde la recolección de información ocurrió en un solo momento específico, por lo que el contexto en que emergen dichos datos corresponde a una similitud legal, histórica y procedimental.

De este modo, la técnica de investigación seleccionada correspondió al análisis de contenido, estrategia metodológica que consiste en el estudio de la realidad social por medio de la observación directa y/o por medio de análisis de documentos y/o datos secundarios que se producen, con un objetivo 0 finalidad distinto del que se propone quien pretende utilizarlos (González, 1994; López-Aranguren, 1994). En este estudio en particular, se utilizó el análisis de contenido por medio de documentos 0 datos existentes de tipo escrito correspondientes en informes periciales psicológicos respecto de niños, niñas y adolescentes que han sido víctimas de delitos sexuales por parte de un agresor menor de 18 años. De este modo, al utilizar datos secundarios, se evitó la revictimización de los sujetos que se pretenden analizar, dado que debieron encontrarse o se mantienen en un proceso de diligencias de investigación que en muchos casos es maltratante y/o vulnerador (Oficina de Defensoría de los Derechos de Infancia, 2006). Por lo que se buscó cumplir con el resguardo ético de "resguardar el bienestar y los derechos de las personas participantes" que resguarda el Colegio de Psicólogos de Chile (1999, Pág. 14).

Esta elección metodológica se funda, por otro lado, en la calidad y credibilidad de los datos existentes escogidos, los que corresponden a una institucionalidad específica, Corporación Opción, organismo que cuenta con lineamientos básicos y mínimos del contenido de sus peritajes (Opción, 2004), además de contar con el reconocimiento del Estado (SENAME), dado que su funcionamiento se encuentra supeditado a la licitación por parte de ésta.

Los informes periciales psicológicos pertenecen al Programa Ambulatorio de Independencia (DAM Independencia), línea 
programática que aborda situaciones de vulneraciones de derechos de niños, niñas y adolescentes menores de 18 años, efectuando informes periciales tanto proteccionales como penales, derivados de solicitudes del sistema de justicia (Tribunales de Familia, SENAME y Ministerio Público). El programa del que fueron obtenidos los informes comprende la territorialidad de las siguientes comunas de la Región Metropolitana: Independencia, Huechuraba, Quilicura, Renca, Recoleta, Colina, Lampa y Til Til.

A su vez, los informes obtenidos comprendían los períodos entre los años 2008-2010, archivos que se encontraban completos y el contexto de su construcción no posee características disimiles relevantes, mientras que la variabilidad de profesionales evaluadores fue de 9 psicólogos con especialización en temáticas de psicología forense, permitiendo la disminución de sesgo de emisión de la información ante la ampliación del espectro de evaluadores.

De este modo, el muestreo correspondió a un muestreo no probabilístico, puesto que se desconoce la probabilidad que tienen los elementos de la población de ser seleccionados para conformar la muestra, especialmente si se considera la alta cifra negra en dicho tipo de delitos (Escaff y Salinas, 2005). Por lo que la muestra fue definida arbitrariamente y en función de criterios teórico-prácticos, siendo de este modo intencionado, donde finalmente fueron recolectados 76 informes tanto proteccionales como penales, distribuyéndose en 45 y 31 respectivamente.

La totalidad de los informes cumplían los criterios de inclusión definidos para la presente investigación, los que corresponden a:
- Niños, niñas y adolescentes que hayan sufrido una victimización sexual cometida por un agresor menor de 18 años.

- Casos que se encuentren judicializados por el sistema de justicia proteccional $y / 0$ penal chileno.

- Víctimas que hayan sido evaluados psicológicamente por el Programa de Diagnóstico Ambulatorio de Independencia, tanto en las modalidades de Pericia Proteccional como Penal Psicológica.

Las variables consideradas en el presente estudio corresponden a dinámica abusiva, componentes de vulnerabilidad y estadios del desarrollo evolutivo, los que se desprenden del problema, pregunta y objetivos de investigación antes señalados. La variable de dinámica abusiva corresponde a la relación y situación de agresión sexual mantenida entre víctima y victimario, donde se desprenden las subcategorías: vínculo entre víctima $\mathrm{y}$ victimario, forma de sometimiento, conducta de transgresión sexual ejercida, frecuencia, y develación y denuncia.

En relación a los componentes de vulnerabilidad esta variable corresponde a la condición personal, familiar, o grupal que favorecen instancias de asimetría de entre sujetos, o en el caso victimológico; de la víctima para con su victimario. De esta variable se desprenden las subvariables: características personales y sociofamiliares. Y por último, de los estadios del desarrollo evolutivo, si bien podría ser considerada como un elemento de características personales, fue definida como una característica que se evaluó a parte, debido la profundidad de la misma y la asociación de variables que se pretendió indagar. Así, se estiman 4 grupos de desarrollo infantojuvenil que fueron definidos para la presente investigación, a partir de una homogeneización respecto de los diversos ámbitos que componen el desarrollo 
evolutivo (cognitivo, social, moral, sexual y afectivo) desde una agrupación teórica de las capacidades atribuibles a dichos rangos etarios, los que corresponden a: primera infancia y preescolaridad (2-5 años), infancia o niñez (6-8 años), preadolescencia (9-13 años) y adolescencia (14-17 años).

\section{RESULTADOS}

\section{Componentes de vulnerabilidad}

Dentro de los componentes de vulnerabilidad explorados se encuentran las características personales y sociofamiliares de las víctimas, cabe destacar que en todos los casos revisados existe co-existencia de factores de vulnerabilidad. Respecto del primer sub-grupo, es posible mencionar que en la revisión de documentos se encuentra información respecto de todos los elementos de dicha categoría.

En relación al sexo de las víctimas, de los 76 documentos, que hacen alusión a una víctima en particular, 41 de estas corresponden a mujeres, mientras que las 35 restantes son de sexo masculino, denotándose una leve tendencia superior en aquellas víctimas de sexo femenino (Figura. 1).

\section{FIGURA 1.}

Sexo de las víctimas infanto-juveniles de agresores sexuales cometidos por otro niño, niña y/o adolescente.

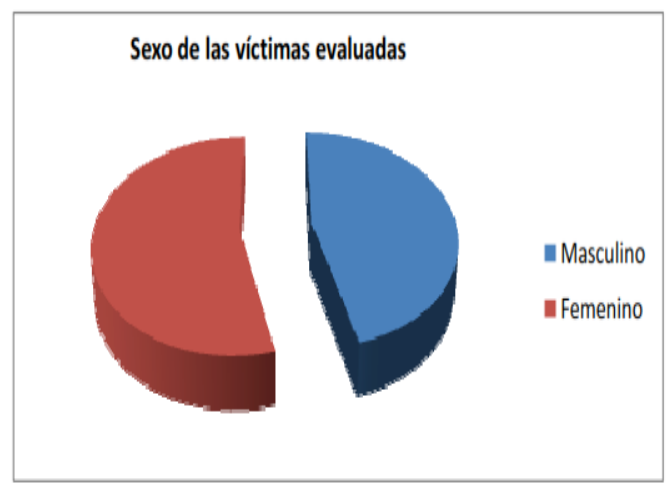

Respecto de la edad de las víctimas peritadas, la mayor cantidad se concentra en la edad de la niñez y/o infancia, correspondiente al rango de los 6 a 8 años de edad, agrupándose en 42 víctimas. De este modo, se encuentra consecutivamente víctimas que se hallan en edad preescolar, correspondiente a 22 de los peritados del total de documentos consultados. Mientras que de manera homogénea y como una tendencia minoritaria, se encuentran víctimas en los rangos propios de la adolescencia y preadolescencia, con 7 y 5 sujetos respectivamente (Figura 2).

\section{FIGURA 2.}

Rangos etarios de las víctimas infanto-juveniles de agresores sexuales cometidos por otro niño, niña y/o adolescente.

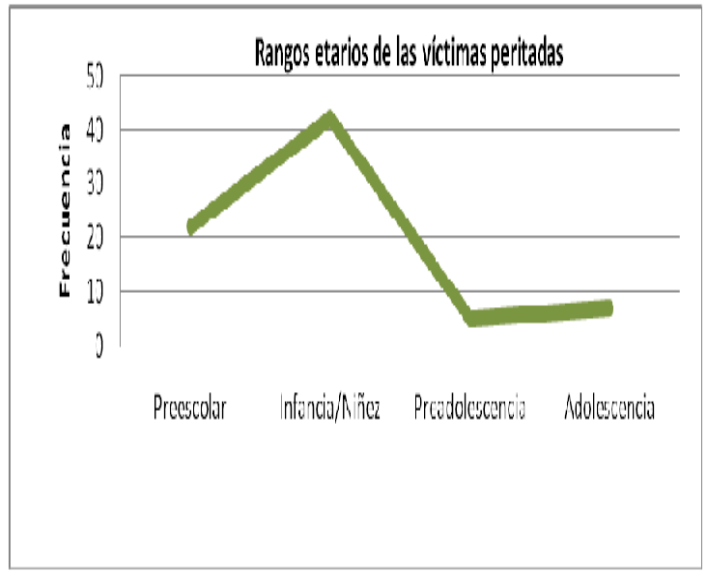

Respecto de la existencia de dificultades en el desarrollo psicomotor, en ninguno de los informes se señala respecto de trastornos en el área, tanto en la capacidad actual como mórbida de las víctimas. Asimismo, en relación a las capacidades cognitivas, sólo en una tendencia minoritaria se destaca la existencia de un desarrollo intelectual menor al esperado en relación a su rango etario, no obstante ninguno de esos casos da cuenta de la existencia de retraso mental y hacen alusión a problemas de aprendizaje, baja estimulación y/o problemas emocionales.

Por otro lado, en relación a las características de personalidad, es importante destacar la emergencia de información consistente a los componentes del desarrollo emocional de las víctimas, donde se hace referencia a carencias 
afectivas, que se relacionan y vincula con otras características observadas como inmadurez emocional, dificultades vinculares y compromisos emocionales producto de conflictos familiares (Figura 3 ).

FIGURA 3.

Componentes del desarrollo emocional.

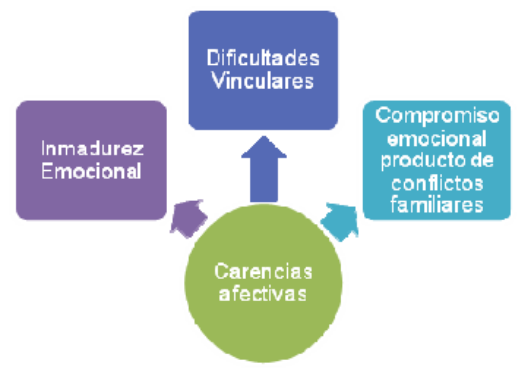

En relación a las características conductuales y relacionales, se observan principalmente dos estilos conductualesrelacionales en los documentos observados, aquellos que propenden a modalidades con mayor pasividad y dependencia, y aquellas de tipo activas-disruptivas. En el primer estilo se encuentran elementos de timidez, introversión/exclusión social, dependencia excesiva; mientras que en el otro se destacan la existencia de déficit atencional, dificultad en acatar normas y límites, y dominancia (Figura 4).

FIGURA 4.

Características conductuales y relacionales.

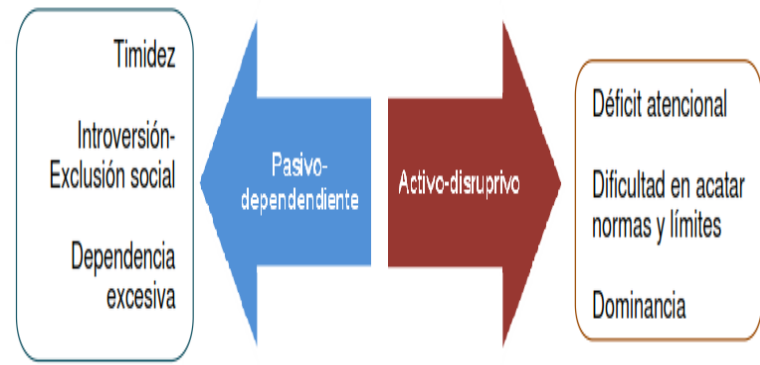

Por otro lado, al explorar la composición biográfica de la víctima, excluyéndose hitos del continuo vital de índole familiar que son indagados en otra sub-categoría, se pueden destacar que en gran parte de los documentos se consignaba la inexistencia de antecedentes mórbidos. No obstante en aquellas víctimas que si poseían dicho antecedente, se mencionaron la existencia de complicaciones gestacionales y/o neonatales, problemas de control de esfínteres, trastornos alimenticios y antecedentes de agresión sexual previa (Figura 5).

\section{FIGURA 5.}

Antecedentes mórbidos de niños/as y adolescentes víctimas de agresiones sexuales.

\section{Antecedentesmórbidos}

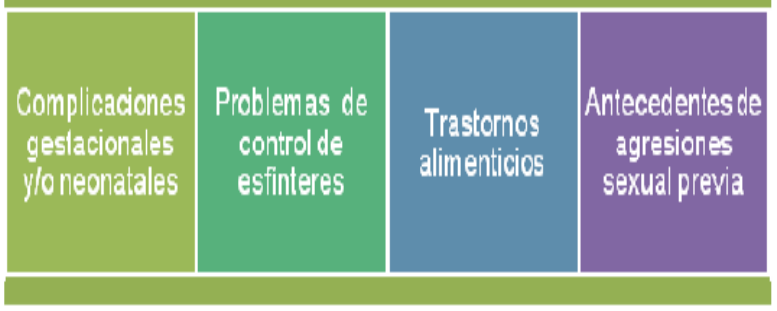

De las características socio-familiares de la víctima, los antecedentes referentes a la estructura familiar presente en los informes consultados, se destaca la existencia de continuos cambios a nivel de su estructura a lo largo de, en ocasiones, breves lapsos de tiempo. No obstante, fue posible destacar la existencia de una predominancia en torno a las familias de tipo extensa, nuclear y reconstituida, encontrándose con menor tendencia aquellas organizaciones exclusivamente monoparental y con sustitución de rol. Especialmente dentro de esta última, se encuentran bajos casos de institucionalización del niño, niña y/o adolescente, aspecto que podría deberse a la naturaleza de los documentos analizados, dado que corresponden a un proyecto de carácter ambulatorio y dicha información podría encontrarse con mayor prevalencia en programas de diagnóstico residencial.

Por otro lado, en relación a las dinámicas familiares es posible señalar la existencia de tendencias similares a la señalada en la 
literatura especializada en referencia a vulnerabilidad sociofamiliar en delitos sexuales contra niños, niñas y adolescentes. Dichas categorías corresponden a: ausencia del padre, violencia intrafamiliar, ausencia de la madre, conflicto entre los miembros de la familia, y dificultad en el establecimiento de límites y normas. A su vez, emergen los siguientes dos elementos: negligencia parental $y$ externalización de cuidados de la víctima en terceros, los cuales mantienen una importancia relevancia y presencia respecto de la totalidad de documentos analizados. Asimismo, se observa en menor grado, información respecto de presencia de alcoholismo y drogas, precariedad económica y abandono (Figura 6).

FIGURA 6.

Características de dinámicas familiares.
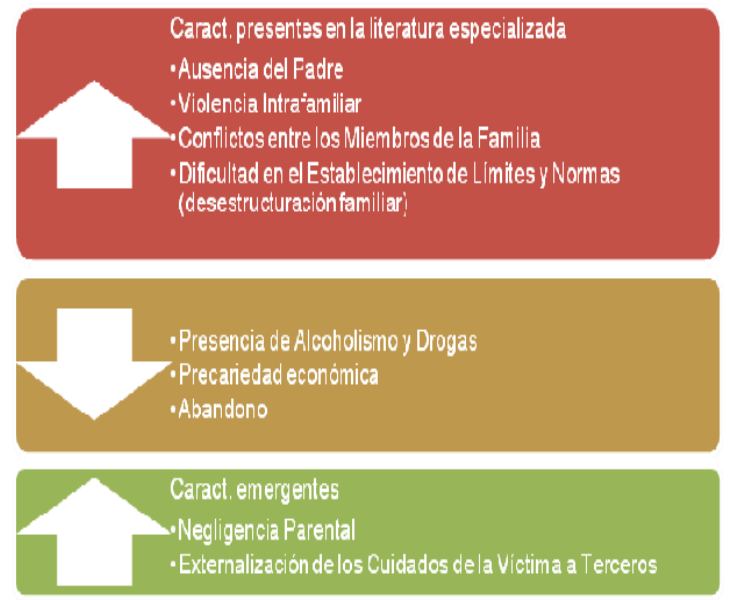

\section{Dinámica abusiva}

En relación a los resultados obtenidos respecto de la dinámica abusiva y los elementos que la componen, es posible señalar que dentro del vínculo entre víctima y victimario, solamente se encuentran casos donde existe algún grado de relación previa entre estos, sin encontrarse informes donde exista un agresor desconocido. De este modo, fue posible evidenciar diversas formas de vínculos de carácter conocido e intrafamiliar, donde la tendencia entre ambos es similar, no existiendo una mayor y/o significativa prevalencia de uno por sobre el otro (Figura 7).

FIGURA 7.

Tipos de vínculos existentes en agresiones sexuales entre víctimas y victimarios infantojuveniles.

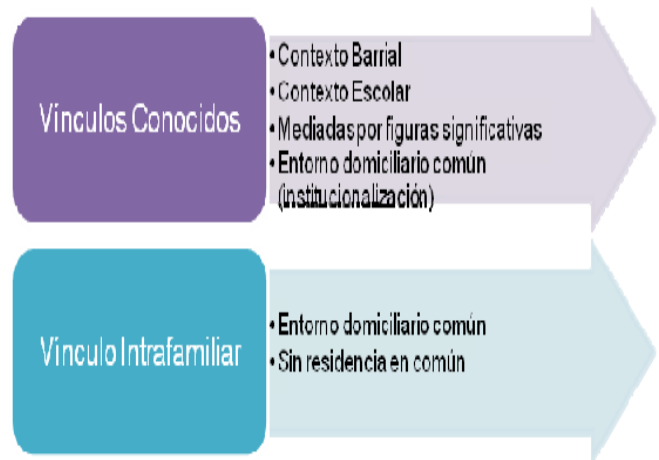

Otra categoría que se desprende de la dinámica abusiva, corresponde a la forma de sometimiento existente en la situación de agresión sexual. A partir de la revisión realizada es posible constatar la coexistencia de diversas formas de sometimiento, específicamente a partir de los diversos objetivos que dichos mecanismos poseen dentro de la dinámica abusiva: la posibilidad de perpetración del hecho abusivo y la evitación de una eventual develación.

De este modo, los documentos consultados entregan información respecto de transgresiones por medio de la incredulidad, uso de violencia física, existencia de amenazas, manipulación, engaño y utilización de dinero, respectivamente en términos de reiteración de los elementos (Figura 8). Así, es posible señalar que existe una mayor tendencia respecto del uso de métodos de coacción implícita, no obstante en variados casos se observa la presencia de ambas formas tanto explícitas como implícitas del uso de la violencia como forma de lograr una asimetría relacional entre víctima y victimario. 
FIGURA 8.

Uso de formas de sometimiento en virtud de objetivo.

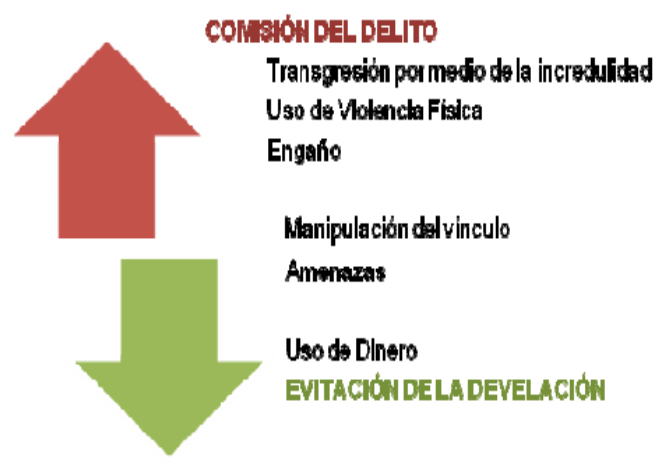

Por otro lado al considerar, dichos elementos en términos del grado de cercanía y/o dependencia que se establece entre víctima y victimario, es posible señalar que mayor relación de horizontalidad y/o asimetría se observa una mayor tendencia de manipulación del vínculo, engaño y uso de dinero por parte del agresor (Figura 9). Por tanto, en situaciones en que el vínculo es más precario y/o casi inexistente por que se encuentra mediado por terceros, se visualiza una mayor transgresión por medio de la incredulidad y uso de violencia física. De este modo, las amenazas aparentemente no se encuentran con una supeditación a ninguno de los dos polos.

FIGURA 9.

Uso de formas de sometimiento en virtud de la cercanía vincular.

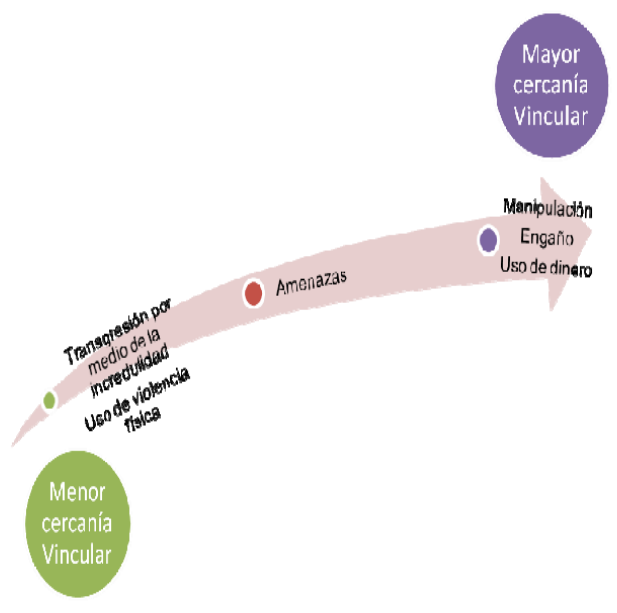

Por otro lado, dentro de la sub-categoría de las conductas de transgresión sexual ejercida, es posible señalar que dentro de los documentos revisados se encontraban tendencias similares respecto de actos penetrativos de índole oral, vaginal y anal, y aquellos de tipo no penetrativo consistente en tocaciones. Solamente en un caso, se señala la realización de fotografías por parte del agresor y exposición a material pornográfico, no obstante esta situación se consigna dentro un contexto de juegos de connotación sexual, donde habría existido una variabilidad de actos sexuales consistente en penetraciones y tocaciones.

Otra sub-categoría revisada corresponde a la frecuencia de victimización, elemento donde se evidenció una mayor tendencia de frecuencias sistemáticas, encontrándose consecutivamente eventos únicos. Solamente en una oportunidad, existió información que podría encontrarse dando cuenta de una frecuencia de carácter crónico, pero que no obstante no se encuentra consignado explícitamente de dicha forma dentro del informe, si no que al momento de explicar el daño de dicha víctima.

De este modo, los elementos que permiten configurar esta sub-categoría será similar en todas las frecuencias, por tanto situaciones donde existe una mayor cercanía física y/o vínculo afectivo, no sólo permitirá mayores contextos de posibilidad sino también respecto de la utilización de formas de sometimiento menos disruptivas que permiten que la dinámica abusiva se instale en las pautas de interacción entre víctima-victimario (Figura 10).

Y como última sub-categoría de la dinámica abusiva, la develación y denuncia son elementos que emergen en los documentos analizados, y donde se observa la existencia de cierta variabilidad, aunque predominan aquellas develaciones de tipo circunstancial y/o accidentales en que la víctima no tiene motivación para que la 
situación abusiva sea conocida por sus figuras significativas. Asimismo, a pesar de la propensión de develaciones de este tipo, se observa una mayor tendencia a una denuncia inmediata por parte de los adultos que se encuentran al cuidado de las víctimas de dicho tipo de delito.

FIGURA 10.

Elementos relacionados con la frecuencia de victimización.

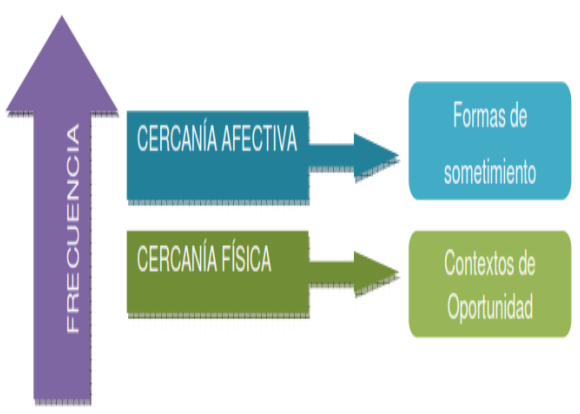

De este modo, se pueden visualizar que dichas develaciones de carácter circunstancial, tienden a ocurrir a partir de las presiones por parte de las figuras parentales a raíz de: cambios conductuales y/o emocionales, evidencias y/o daño físico y/o develaciones de terceros (Figura 11).

FIGURA 11

Elementos favorecedores de una develación circunstancial.

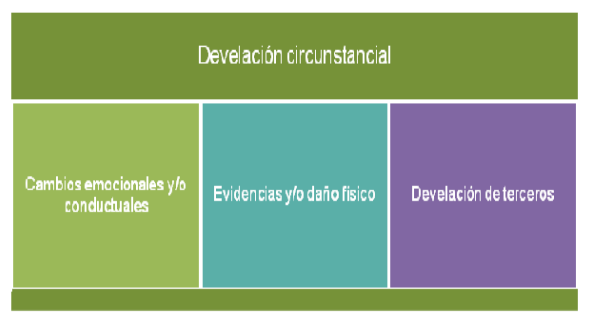

De este modo, la develación y denuncia corresponderá y reflejará aspectos de la funcionalidad del sistema familiar, en términos de la capacidad y/o competencias parentales de las figuras significativas de ese niño, niña y/o adolescente en términos de captar señales que dan cuenta de una situación anómala y por ende responder ante dicho contexto en virtud de restituir las adecuadas condiciones para el bienestar de la víctima.

Asimismo, se observa una mayor prontitud en el establecimiento de la denuncia, cuando el imputado corresponde a un contexto extrafamiliar. Igualmente, la existencia de una mayor tendencia de develaciones de carácter circunstancial es posible de explicar y/o relacionar, en ocasiones, ante la imposibilidad de comprensión por parte de las víctimas, a veces por su corta edad, de la noción de transgresión y la ilicitud de dicha conducta. La cual no sólo se adscribe a los tramos etarios en que tanto víctima como victimario se encuentran, sino también ante la existencia de un vínculo de simetría que se comparte.

Estadios del desarrollo evolutivo en víctimas y agresores

En relación a los estadios del desarrollo en víctimas y agresores sexuales menores de edad, es importante destacar que en algunos de los informes revisados, no se consignaba el rango etario ni la edad específica del ofensor. No obstante, en todos los documentos utilizados, se explicitaba que el responsable de dichos actos fuese menor de edad. Por otra parte, se destaca que en ninguno de los casos revisados se consigna la existencia de una víctima de mayor edad en relación a su agresor.

De este modo, sólo se encuentran dos tendencias; que la víctima mantuviese una edad menor que el agresor y/o que ambos se tuvieran la misma edad. Esta última configuración, también se observa minoritaria ante aquellos casos donde el agresor es mayor a su víctima. Así, los rangos etarios de las víctimas en términos de mayor prevalencia corresponden a los estadios de la niñez, preescolaridad, adolescencia y preadolescencia. Mientras que en los agresores, existe una importante tendencia a concentrarse en la 
adolescencia, 41 casos, y posteriormente posicionarse decrecientemente en los rangos etarios de la preadolescencia, niñez y preescolaridad con 16, 6 y 3 casos respectivamente (Figura 12).

FIGURA 12.

Estadios del desarrollo de los agresores infantojuveniles.

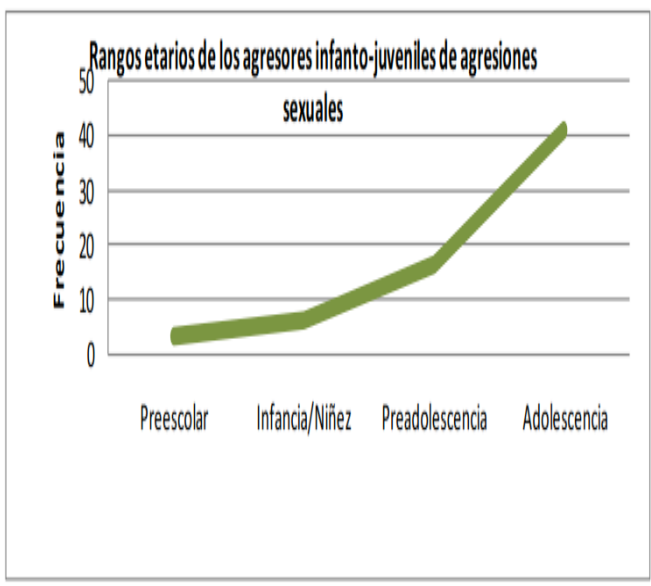

Asimismo, es importante destacar que todos los sujetos consignados como agresores corresponden al sexo masculino, y solamente en uno de los casos se hace mención a conductas de externalización en una víctima de sexo femenino, quien tendía a replicar las conductas abusivas con un hermano de menor edad.

Por otro lado, las diferencias etarias entre víctima y victimario mantuvieron gran heterogeneidad, aunque es posible observar una predominancia respecto de aquellos casos en que existía una diferencia de 7 años en relación a los otros casos. Por lo que los rangos de diferencias etarias se encontraba entre los 14 años de diferencia y los 0 años. A partir de lo anterior, es posible señalar que en los rangos etarios asociados a dichas diferencias etarias, sólo se encontraron 11 casos en que tanto víctima y victimario compartía una misma etapa evolutiva similar (Tabla 1).
TABLA 1.

Diferencias de edad entre víctimas y victimario.

\begin{tabular}{llllllllllllllll}
\hline Dïerercia & 14 & 13 & 12 & 11 & 10 & 9 & 8 & 7 & 6 & 5 & 4 & 3 & 2 & 1 & 0
\end{tabular}

Frecuencia

Dinámicas de agresión sexual a partir del desarrollo evolutivo de víctima y victimario

A partir de lo anterior, solamente fue posible encontrar dos grupos en torno a este fenómeno en relación al desarrollo evolutivo, cuando existe un nivel evolutivo dispar entre víctima y victimario, o cuando es coincidente.

Respecto del primer grupo, es posible señalar que estos se encuentran en un contexto de ocurrencia pertenecientes al contexto escolar y barrial de la víctima. En esta configuración, existe mayor proporción de casos donde la cercanía emocional no corresponde a un elemento significativo de la relación, por lo que las formas de sometimiento en dicho tipo de contexto corresponden a mecanismos, en mayor medida, de coerción explícita, consistente en uso de violencia y/o amenazas. Esto último da cuenta, de la importancia de este mecanismo en virtud la posibilidad de perpetrar el acto, más que la relevancia en la mantención de esta dinámica en el tiempo.

Lo anterior puede deberse a que esta configuración del fenómeno se encuentra en víctimas y victimarios que se encuentran en un rango evolutivo de la niñez, por lo que el desarrollo evolutivo en términos morales, la representación de las consecuencias por parte del niño o su descubrimiento en el futuro se encuentran disminuidas, por lo que no se privilegiaría mecanismos de sometimiento en relación a la evitación de la develación. En esta línea, ante la existencia un desarrollo evolutivo incipiente en términos sociales y cognitivos, el uso de la violencia como mecanismo de control podría 
posicionarse como un método validado desde el victimario como su víctima, ya que el desarrollo de una perspectiva relacional diferenciada es menor, y por ende, mecanismos más sofisticados no son siquiera pensados.

En términos de la frecuencia, las agresiones sexuales son de episodio único y son develados en mayor medida de manera espontánea y oportuna, a diferencia de casos donde existe antecedentes previos de abuso por parte de otro agresor.

En relación a los componentes de vulnerabilidad, es posible señalar la existencia de alta variabilidad y coexistencia de estos, los que se diferenciarán por cercanía vincular. Por tanto, dentro del contexto barrial, a partir un lazo de amistad, se observa una menor cantidad de componentes de vulnerabilidad, los que tenderán a concentrarse en elementos de vulnerabilidad personal, específicamente de carácter emocional. Mientras que en casos de menor cercanía vincular, como el contexto escolar, se visualizan características personales consistentes en antecedentes precios de vulneración sexual donde no existiría respuesta protectora ni reparatoria de las figuras significativas, y dentro de los aspectos sociofamiliares, se visualizan carencias de acciones de promoción del desarrollo integral de la víctima.

$Y$ respecto del segundo grupo, donde existe disparidad evolutiva entre víctima y victimario, podemos destacar dos grupos dinámicas de agresión sexual intrafamiliar y por conocido. La primera corresponde a un grupo relevante en la presente investigación, donde particularmente se relevan componentes de vulnerabilidad de índole socio-familiar, en términos de dinámicas familiares con mayor disfuncionalidad, como los siguientes: ausencia voluntaria o involuntaria del padre, la que puede ser por medio de negligencia de dicha figura o física, y dificultad de establecimiento de límites y normas.

Este último componente de
vulnerabilidad puede apoyarnos en comprender el contexto de desarrollo psicosocial de tanto víctimas como agresores, donde la imposibilidad de integrar ciertas pautas de interacción claras y con límites, también dificultan el desarrollo progresivo de tipo moral, social y emocional. Esto significa, la posibilidad de concebir a otro como un ente diferenciado y la adquisición de empatía, lo que se define ante la posibilidad de integrar límites personales que desarrollen la propia identidad, elemento que podría relacionarse al modelo de Wolf (1988) respecto de uno de los factores de las experiencias tempranas que desarrollan una personalidad con predisposición a configurar intereses sexuales desviados.

Por último, dentro de las dinámicas de agresión sexual por parte de un conocido con desarrollo evolutivo dispar en relación a su víctima, es posible señalar la alta variabilidad $y$ heterogeneidad en las diferencias de vínculo entre víctimavictimario. No obstante, es importante destacar que el mayor desarrollo evolutivo en el agresor se establecerá como una ventaja en términos de la capacidad de configurar los elementos necesarios para perpetrar y mantener en secreto la dinámica abusiva. Sin embargo, la mera disparidad evolutiva no es suficiente, muy por el contrario, incluso en aquellos casos con alta diferencia evolutiva entre víctima-victimario, siempre fue posible constatar algún componente de vulnerabilidad por parte de la víctima. Lo que podría corresponder a un aprendizaje paulatino por parte de este en torno al desarrollo de la capacidad de visualizar la factibilidad de transgresión en una víctima específica, ya sea por la posibilidad de perpetración como por impedir una eventual develación. Esto también se relaciona con la variabilidad de formas de sometimiento con diferentes 
fines, los que posibilitan que se establezca una dinámica de asimetría.

\section{DISCUSIÓN}

El fenómeno de las agresiones sexuales tanto infanto-juveniles como aquellas que se cometen a adultos, especialmente a mujeres, requieren de un mayor análisis en términos de la precisión de los contextos de ocurrencia en que estas ocurren. Es posible evidenciar en la literatura especializada, que la búsqueda por indicadores de agresión sexual, siempre será vana e inútil incluso, si no distinguimos ciertas variables que diferenciarán la configuración de esta forma específica de violencia.

En relación a las diferencias y similitudes con estudios en el área, especialmente aquellos de tipo internacional, es posible constatar que existe una coincidencia en relación al vínculo a la base de la dinámica abusiva infanto-juvenil, donde existe una mayor proporción de índole intrafamiliar. No obstante, el promedio de edad de los agresores no fue coincidente entre la presente investigación y el trabajo de Johnson (1988), existiendo un promedio de edad del agresor asociado a la adolescencia a diferencia del otro trabajo donde existe una predominancia en la niñez. Esto podría deberse a diferencias en los criterios de inclusión y/o en las diferencias culturales que existen a la base para que se visibilice el fenómeno y que se logre la intervención institucional.

En la misma línea, ante la información adquirida por medio de estudios con agresores adultos, donde se señala el inicio de las conductas de transgresión antes de los 16 años, es posible hipotetizar que, si bien existe un aumento de denuncias de delitos sexuales cometidos por niños, niñas y adolescentes, podría existir igualmente un alto número de cifra negra de este fenómeno.
Por otro lado, también pudo ser observado el fenómeno que plantea el estudio de Johnson (1988) de la multiplicidad de víctimas por parte de un mismo agresor. Dicho dato fue posible de recogerlo mediante la develación por parte de terceros, donde a partir de la develación de una víctima es posible constatar la existencia de otras, y a su vez en casos donde el vínculo es de carácter intrafamiliar, se visualizó tanto multiplicidad de agresores como víctimas dentro del mismo contexto intrafamiliar. No obstante, no fue posible acceder a un dato que permitiera conocer el promedio de víctimas por parte del agresor, puesto que no correspondía a los objetivos del presente estudio y no correspondía a un dato abordado en los documentos revisados.

La indagación en relación a componentes de vulnerabilidad y dinámica abusiva es un elemento enriquecedor para poder elaborar o integrar a intervenciones de carácter preventivas, reparatorias y diagnósticas. En esta misma línea, la intervención preventiva en características de vulnerabilidad y/o promoción de desarrollos integrales en niños/as y adolescentes por medio de políticas públicas de infancia, se vuelve vital para favorecer una prevención en torno de situaciones delictuales, como las agresiones sexuales, pero también se torna relevante como medio para favorecer el desarrollo de procesos de resiliencia tras situaciones traumáticas como pueden serlo las transgresiones en la esfera de la sexualidad. La idea de prevención por tanto, no corresponderá sólo a una lógica consistente en disminuir la vulnerabilidad en una potencial víctima, sino que también en reducir factores que permitan que se configure, tal como señala Wolf (1988), el potencial de desarrollo de un tipo particular de personalidad, donde se instaura un interés sexual desviado por parte del potencial agresor.

De esta manera, no podemos conocer el momento en que una vulneración de 
derechos a un niño, niña o adolescente se transforma en un componente de vulnerabilidad tanto para padecer como para ejecutar y cometer a futuro una acción delictual. Si consideramos entonces, el alto número de este tipo de agresiones sexuales de índole intrafamiliar con vínculo fraterno, existe entonces importantes similitudes del contexto de desarrollo tanto de dicha víctima como de dicho agresor. Por lo cual, sería interesante indagar con mayor profundidad en las diferencias y similitudes en términos de las historias de vida que se configuran tanto por parte de dicha víctima como por su agresor, lo que podría permitir conocer elementos diferenciadores para la prevención e intervención/rehabilitación.

La relevancia de conocer elementos del sistema familiar, vinculares, relacionales y del propio desarrollo de agresores infantojuveniles que cometen algún tipo de acción delictual, especialmente aquellos en la esfera de la sexualidad, será especialmente ante lo que señala Boris Cyrulnik (2003) en relación a que este tipo de conductas delictuales no corresponden a una "delincuencia de supervivencia", la cual ha sido la explicación histórica y frecuente a la delincuencia donde se destacan y se enfoca en las condiciones de pobreza, especialmente respecto de aquellos delitos en contra de la propiedad. Muy por el contrario, las agresiones sexuales, para este autor, corresponderían a "delitos de placer", "una especie de deporte de riesgo" (Cyrulnik, 2003, Pág. 181), la que podría deberse a una vinculación y socialización precaria, donde se intenta "expulsar fuera de uno mismo la violencia que no ha sido estructurada por el entorno" (Cyrulnik, 2003, Pág. 180).

De este modo, la observación de que la vulnerabilidad emergerían de elementos de la dinámica familiar, más que de la estructura en sí misma, no es trivial y apunta justamente a lo señalado con anterioridad. Por tanto, la preocupación en torno a las políticas públicas debería encontrarse a la base de fortalecer sus pautas de interacción y no necesariamente sus estructuras, donde se han enfocado múltiples políticas públicas de protección social, a pesar de la diversificación de patrones familiares con la globalización.

Por otro lado, es necesario indagar mayormente en términos de otros aspectos que diversifican el desarrollo evolutivo, a partir de las pautas de socialización diferenciadas, como lo son las perspectivas y construcciones de género. Tal como se esbozaba con anterioridad al momento de señalar la necesidad de explorar mayormente en las historias de vida diferenciados de víctimas como agresores, las construcciones de género podrían corresponder una temática importante de explorar en su vinculación al desarrollo de un potencial de personalidad con un interés sexual desviado, especialmente ante el hecho de que quienes son consignados como agresores sexuales corresponden usualmente al género masculino en los diversos estudios en la materia, y especialmente en esta investigación, solamente niños y adolescentes hombres fueron consignados como transgresores de la sexualidad de otro niño, niña y/o adolescente. $\mathrm{Si}$ asimilamos así $\mathrm{y}$ recontextualizamos los planteamientos de Simon de Beauvoir (1981) que señala "una mujer no nace, se hace", al parecer no sólo victimario si no también su víctima se construye a partir de diversos factores, donde uno de estos puede corresponder al género más que al sexo.

De esta manera, sería relevante explorar en torno de los aspectos en común y diferenciadores de las dinámicas abusivas en relación al género, donde probablemente elementos como la forma de sometimiento y daño podrían entregar características específicas, que permitirían establecer líneas importantes para el diseño de estrategias de terapéuticas. Especialmente si consideramos la interrelación del daño con formas de sometimiento que podrían apelar a manipulaciones que apuntan 
elementos del desarrollo moral femenino, tal como se consignan en los desarrollos teóricos de Gilligan (1994). En la misma línea, sería interesante indagar respecto de la perspectiva de género a la que se adscriben tanto víctimas como victimario, especialmente si consideramos los cambios culturales que han permitido un paulatino aunque leve cambio social en torno de los elementos de género asociados a características sexuales del sujeto.

Asimismo, a partir de la inexistencia de casos encontrados en esta investigación en torno a agresores de sexo femenino, nos permite preguntar respecto al proceso en que se desarrollan las conductas de victimización en ofensoras mujeres en relación a su edad y etapa de desarrollo. Particularmente, porque en el caso de agresores de sexo masculino, gran parte de las conductas de transgresión sexual comienzan justamente en los estadios del desarrollo que esta investigación analizó.

En relación a las reflexiones que pueden emerger de este estudio como aporte a las líneas de intervención, es posible destacar que en los ejes de protección, superación de la situación de crisis y re-elaboración de la situación abusiva, la noción de que una situación de transgresión sexual por un niño, niña y/o adolescente mantiene una configuración muy similar en ocasiones a la que podría tener una agresión sexual cometida por un adulto, ya que esta no tiene necesariamente una satisfacción sexual sino de poder sobre otro. Por lo que dicho tipo de situaciones no pueden ser asimiladas a la ligera como "juegos de niños".

En el caso de las agresiones sexuales intrafamiliares, sería necesaria el desarrollo de cambios en las pautas de relación que facilitan la victimización, donde la restitución de los roles adecuados en las figuras significativas o de apego subsidiaria es vital, al igual que la indagación y corrección de elementos de negligencia parental, especialmente respecto de no sólo la adopción de medidas de protección al niño, niña o adolescente que es víctima, sino que también en relación al establecimiento de pautas de crianza y favorecimiento de desarrollo integral. Esto se debe a que usualmente la reconfiguración familiar tras la victimización, tendrá asociada la necesidad de plantearse la exclusión 0 integración del agresor en el grupo familiar.

Igualmente, la importancia del vínculo como facultativo para la consecución de la transgresión sexual, corresponderá también a un elemento importante al momento de la superación de la situación de crisis y su eventual re-elaboración de la situación abusiva, puesto que los vínculos de confianza se encontrarán en cuestionamiento, y muchas veces, justamente en aquellos que se configuran dentro del grupo identitario común; los pares y/o la familia.

Por otro lado, en términos diagnósticos, los elementos diferenciadores respecto de los distintos tipos de vínculo que en la presente investigación emergen, permiten establecer hipótesis de exploración en términos de las diferentes configuraciones de las dinámicas de agresión sexual cometidas por otro niño, niña o adolescente. Asimismo, la búsqueda de factores de vulnerabilidad que habrían facilitado la victimización, podría ser útil al momento de ligar la situación de vulneración con la transgresión sexual como un elemento facilitador del que podría darse cuenta eventualmente a los Tribunales de Familia por medio de un informe pericial de índole proteccional.

Es importante entonces, el desarrollo de la investigación en este fenómeno, pero también que refuercen y retroalimenten los informes periciales con este tipo de información, especialmente ante la emergencia del dato en relación a la existencia de mayor número de denuncias directas, lo que se condice con las estadísticas chilenas y generales en torno al aumento de denuncias. Si bien esto podría 
corresponder a una mayor confianza de la ciudadanía en relación a la respuesta estatal de la resolución de conflictos, es necesario tener entonces una efectiva solución ante este fenómeno.

No obstante para eso, es necesaria la mayor exploración y análisis del fenómeno, principalmente ante la dificultad que existe por parte de los adultos en general, para asimilar la posible existencia de una conducta de agresión sexual por parte de un niño 0 niña, especialmente por parte de aquellos que se encuentran en etapas tempranas de su desarrollo. Por lo que es necesario integrar una concepción de integralidad en las estrategias y políticas públicas, que no sólo apelen a la disminución de prevalencia de agresiones sexuales, sino que también a la construcción de una mejor sociedad y humanidad.

\section{REFERENCIAS}

Asenjo, F. (2003). Algunos modelos de comprensión de la agresión sexual. En Escaff, E. y Maffioletti, F. (Ed.) Psicología Jurídica: Aproximaciones desde la experiencia (Pp. 235-247). Santiago de Chile: Ediciones Universidad Diego Portales.

Colegio de Psicólogos de Chile (1999). Código de Ética Profesional. Extraído el 5 de Abril de 2011, de: http://www.colegiopsicologos.cl/wpcontent/uploads/2009/08/codigo-etica2.pdf

Correa, A., Navarrete, M. y Salinas, F. (2009). Agresores sexuales adolescentes: Programas de atención e implicancias. En Capella, C., Núñez, L. y Navarro, C. (Comp.) Agresiones sexuales: Reflexiones acerca de las intervenciones psicológicas (Tomo 2) (Pp. 183-211). Santiago de Chile: Universidad de Chile.

Cyrulnik, B. (2003). El murmullo de los fantasmas: Volver a la vida después de un trauma. Barcelona, España: Editorial Gedisa.

De Beauvoir, S. (1981). El segundo sexo. Madrid: Ediciones Aguilar.

Denzin, N. y Lincoln, Y. (2005). Introduction: The Discipline and Practice of Qualitative Research. En Denzin, N. y Lincoln, Y. (Ed.) The Sage Handbook of Qualitative Research (3era. Edición) (Pp. 1-32). California: Sage Publications Inc.

Díaz, J. (2003). Ofensores sexuales juveniles. Revista de estudios de juventud, Vol. 3 (62), Pp. 93-129.

Esbec, E. y Gómez-Jarabo. G. (2000). El psicólogo forense y la prueba pericial psicológica. Madrid: Edisofer.

Escaff, E. y Salinas, M. (2005) Algunas consideraciones acerca de las agresiones sexuales. En Academia Judicial de Chile (Comp.) Derecho de Familia e Infancia: Seminario Internacional (Pp. 63-75). Santiago de Chile: Autor.

Escaff, E. (2009). Intervención en menores agresores sexuales. [Apuntes] Santiago de Chile: Universidad Diego Portales: Magíster en Intervención Psicojurídica y Forense.

Fiscalía Regional Metropolitana Centro Norte (2010). Tabla de frecuencia de delitos sexuales infantiles cometidos a menores de edad. Documento no publicado. 
Fundación Paz Ciudadana (2011). Balance de la Delincuencia 2010. Extraído el 13 de Mayo de 2011 de: http://www.pazciudadana.cl/docs/pub_201 10516100228.pdf

Gilligan, C. (1994). La moral y la teoría. Psicología del desarrollo femenino. México: Fondo de Cultura Económica.

González, B. (1994). La utilización de los datos disponibles. En García, M., Ibañez, J., y Alvira, F. (Comp.) El análisis de la realidad social. Métodos y técnicas de investigación (Pp. 103- 133). Madrid, España: Alianza Editorial.

Guerra, C. y Canessa, P. (2007). Victimas y factores de vulnerabilidad: Análisis de un caso desde el constructivismo. En Corporación de Asistencia Judicial (Comp.) Atención a Víctimas de Delitos Violentos. Reflexiones desde la práctica (2001-2007) (Pp.117-126). Viña del Mar: RIL Editores.

Herrera, C. y Campero, L. (2002). La vulnerabilidad e invisibilidad de las mujeres ante el VIH/SIDA: constantes y cambios en el tema. Salud Pública Mex., Vol. 44 (6), Pp. 554-564.

Horton, C. (1996). Children who molest other children: the school psychologist's response to the sexually aggressive child. School Psychology Review, Vol. 25 (4), Pp. 540-570.

Johnson, T. (1988). Children who molest other children: Preliminary findings. Child Abuse \& Neglect, Vol. 12 (2), Pp. 219-229.

López-Aranguren, E. (1994). El análisis de contenido. En García, M., Ibañez, J., y Alvira, F. (Comp.) El análisis de la realidad social. Métodos y técnicas de investigación (Pp. 461-491). Madrid: Alianza Editorial.

Oficina de Defensoría de los Derechos de la Infancia (2006). Acciones para evitar la revictimización del niño víctima del delito: Manual para acompañar a niños a través del proceso judicial. México: Autor.

Opción (2004). Propuesta de Formatos de Calificación Pericial Psicológica y Social; Reflexiones y Comentarios en torno al tema. Santiago de Chile: Autor.

Salinas, M. (2006). Contextos de Ocurrencia de la Victimización Sexual: Relevancia Diagnóstica. Argentina: Lerner Editorial SRL.

Venegas, R. (2006). Seminario Reflexiones y Experiencias en el trabajo con adolescentes que han agredido
sexualmente-Exposición de Rodrigo Venegas Cárdena. En ONG Paicabí (Ed.) Documentos de trabajo ONG Paicabí N01 (Pp. 6-10). Santiago de Chile: Autor.

Volbert, R. (2005) Sexuelles Verhalten von Kindern: Normale Entwicklung oder Indikator für sexuellen Missbrauch? En Amann, G. y Wipplinger, R. (Ed.) Sexueller Missbrauch: Überlick zu Forschung, Beratung und Therapie (Pp. 449-465). Tübingen, Alemania: Deutsche Gessellschaft für Verhaltenstherapie.

Wolf, S. (1988) A model of sexual aggression/addiction. En Dailey, D. (Ed.) The sexually unusual: Guide to understanding and helping (Pp. 131-148). Londres, Inglaterra: The Haworth Press. 